einziges Ökobilanz-System ist bisher operativ - die Ökobilanz für Packstoffe. Das BUWAL, und hier massgeblich mein VorVor-Redner Herr Dr. H.-P. Fahrni, hat dies mit der EMPA erarbeitet. Das System wird seit einigen Jahren von Schweizer Grossverteilern (angefangen von der $\mathrm{Mi}$ gros, heute auch viele andere) für die ökologische Optimierung ihrer Verpackung angewendet. Momentan wird das System überarbeitet - ich arbeite dabei mit - und die neue Version wird noch dieses Jahr publiziert.

Die Ökobilanz ermöglicht, die ökologischen Auswirkungen von Packmaterialien (Papier, Aluminium, Kunststoffe, etc.) zu vergleichen. Es wird dabei insbesondere möglich sein, verschiedene Umweltbelastungen - Luftverschmutzung, Abwasserbelastung, Energieverbrauch und feste Abfälle - einheitlich zu beurteilen. Eine Ökobilanz stellt somit eine Bewertung der Stoffflüsse anhand ökologischer Kriterien dar. Praktisch geschieht diese so, dass Umweltbelastungen (z.B. g SO$/ 2 / \mathrm{NO}_{x} / \ldots$ pro hergestelltes Produkt) erfasst und anhand deren ökologischer Schadensbedeutung bewertet werden (vgl. Fig. 4 und die Schriften von Braunschweig, BUWAL und MüllerWenk).

Basis einer Ökobilanz ist damit einerseits die Erfassung der Stoffflüsse, welche mit einem Produkt oder einem Prozess verbunden sind. Die Stoffbilanz stellt eine 'conditio sine qua non' dar im Hinblick auf eine sinnvolle ökologische Unternehmungspolitik. Eine Stoffbilanz, zumindest der zentralen Stoffflüsse, kann von den meisten Unternehmungen bereits heute mit vertretbarem Aufwand erstellt werden, und dies sollte auch getan werden.

Die darauf aufbauende ökologische $\mathrm{Be}$ wertung überfordert jedoch die einzelne Unternehmung zumeist. Hier sind primär wissenschaftliche Stellen gefordert, Eckwerte zur ökologischen Bewertung ('Ökofaktoren') beizusteuern. In der in der BUWAL-Schriftenreihe erscheinenden überarbeiteten Ökobilanz für Packstoffe werden sich erste Ökofaktoren für zentrale Umweltbelastungen finden, welche auch in anderen Branchen verwendet werden können. Eine ökologische Bewertung wird erlauben, die Anstrengungen bezüglich Aufwand (an Zeit und Finanzen) und ökologischem Return-on-Investment zu optimieren. Nur am Rande sei erwähnt, dass Ökobilanzen darüber hinaus eine ökologisch gezielte Führung eines Unternehmens entscheidend erleichtern oder sogar erst ermöglichen werden. Denn eindeutige und einheitliche ökologische Werte erlauben klare Zielvorgaben ('Mondlandung!') über einzelne Produkte hinaus. Auf der Basis einer umfassenden Ökobilanz können dann z.B. 'Umweltbudgets' ins Management by Objectives integriert werden, um nur ein Beispiel zu nennen.
Zusammenfassend gilt m.E., dass

- die effektive Entsorgung nicht unbedingt Aufgabe des Herstellers ist, wohl aber das Entsorgungsdesign eines Produktes;

- die optimale Entsorgbarkeit erreicht werden kann, indem - wie an Beispielen gezeigt - die Produktentwicklung den gesamten Produktlebenszyklus ins ökologische Kalkül einbezieht;

- eine umfassende ökologische Optimierung auch davon abhängt, klare ökologische Ziele setzen zu können. Dazu ist die Weiterentwicklung der Ökobilanz als betriebliches Führungsinstrument notwendig.

A. Braunschweig: Die Ökologische Buchhaltung in der städtischen Umweltpolitik, in: Dokumente und Informationen zur Schweiz. Orts-, Regional- und Landesplanung (DISP) Nr. 97/April 1989, ETH Hönggerberg Zürich, 1989.

BUWAL: Ökobilanzen von Packstoffen. Schriftenreihe Umweltschutz Nr. 24, Bern, 1984.

T. Dyllick: Ökologisch bewusste Unternehmensführung - Der Beitrag der Managementlehre. Ö.B.U.Schriftenreihe 1/1989. St. Gallen, 1989. 32 S.

J. Elkington, T. Burke: Umweltkrise als Chance. Okologische Herausforderung für die Industrie. Zürich, 1989.

R. Müller-Wenk: Die ökologische Buchhaltung. Frankfurt, 1978.

\title{
Reststoffentsorgung bei Kehrichtverbrennungsanlagen
}

\author{
Conrad M. Bader* und Jürg Wiedersheim
}

\section{Einleitung}

Abfall produzieren nicht nur Industrie und Gewerbe, die alles daran setzen, um diesen zu verringern. Abfälle produzieren auch die Haushalte, also wir alle. Und da in der Schweiz glücklicherweise heute $80 \%$ des Kehrichts in einer Kehrichtverbrennungsanlage beseitigt wird, müssen die beträchtlichen Mengen der dort anfallenden Reststoffe umweltverträglich entsorgt werden. Dieser Beitrag stellt die heute dazu bereits kommerziell verfügbaren Verfahren vor.

\section{Was sind die Reststoffe einer Kehrichtverbrennung?}

In der Schweiz stehen heute $36 \mathrm{Keh}$ richtsverbrennungsanlagen in Betrieb, die im Jahre 1988 insgesamt 2300000 t Kehricht entsorgt haben.

Die wesentliche Aufgabe der Kehrichtverbrennung besteht darin:

1. Masse und Volumen der Abfälle zu verringern,

2. durch Oxidation der Abfälle Schadstoffe soweit wie möglich zu zerstören,

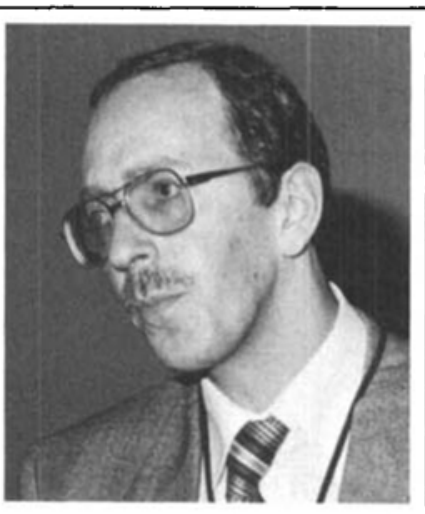

Conrad Bader: Geboren 1952, erwarb 1976 an der ETH Zürich sein Diplom als Maschineningenieur in Fachrichtung Verfahrenstechnik. Nach Tätigkeiten bei den Firmen Escher Wyss, Cora Engineering und Alfa Laval in den Bereichen Prozesstechnik und Marketing trat er 1987 als Assistent Marketing in den Bereich 'Trenn- und Mischverfahren' der Gebrüder Sulzer ein und leitet seit Mitte 1988 die Gruppe 'Umwelttechnik'. Diese entwickelt und baut Sondermüllverbrennungsanlagen, weitergehende Rauchgasreinigungen sowie Aufbereitungsanlagen für Reststoffe von Kehricht- und Sondermüllverbrennungsanlagen.

\footnotetext{
* Korrespondenz: C.M. Bader Gebrüder Sulzer $A G$

Umwelttechnik

Postfach

$\mathrm{CH}-840 \mathrm{l}$ Winterthur
} 
einziges Ökobilanz-System ist bisher operativ - die Ökobilanz für Packstoffe. Das BUWAL, und hier massgeblich mein VorVor-Redner Herr Dr. H.-P. Fahrni, hat dies mit der EMPA erarbeitet. Das System wird seit einigen Jahren von Schweizer Grossverteilern (angefangen von der $\mathrm{Mi}$ gros, heute auch viele andere) für die ökologische Optimierung ihrer Verpackung angewendet. Momentan wird das System überarbeitet - ich arbeite dabei mit - und die neue Version wird noch dieses Jahr publiziert.

Die Ökobilanz ermöglicht, die ökologischen Auswirkungen von Packmaterialien (Papier, Aluminium, Kunststoffe, etc.) zu vergleichen. Es wird dabei insbesondere möglich sein, verschiedene Umweltbelastungen - Luftverschmutzung, Abwasserbelastung, Energieverbrauch und feste Abfälle - einheitlich zu beurteilen. Eine Ökobilanz stellt somit eine Bewertung der Stoffflüsse anhand ökologischer Kriterien dar. Praktisch geschieht diese so, dass Umweltbelastungen (z.B. g SO$/ 2 / \mathrm{NO}_{x} / \ldots$ pro hergestelltes Produkt) erfasst und anhand deren ökologischer Schadensbedeutung bewertet werden (vgl. Fig. 4 und die Schriften von Braunschweig, BUWAL und MüllerWenk).

Basis einer Ökobilanz ist damit einerseits die Erfassung der Stoffflüsse, welche mit einem Produkt oder einem Prozess verbunden sind. Die Stoffbilanz stellt eine 'conditio sine qua non' dar im Hinblick auf eine sinnvolle ökologische Unternehmungspolitik. Eine Stoffbilanz, zumindest der zentralen Stoffflüsse, kann von den meisten Unternehmungen bereits heute mit vertretbarem Aufwand erstellt werden, und dies sollte auch getan werden.

Die darauf aufbauende ökologische $\mathrm{Be}$ wertung überfordert jedoch die einzelne Unternehmung zumeist. Hier sind primär wissenschaftliche Stellen gefordert, Eckwerte zur ökologischen Bewertung ('Ökofaktoren') beizusteuern. In der in der BUWAL-Schriftenreihe erscheinenden überarbeiteten Ökobilanz für Packstoffe werden sich erste Ökofaktoren für zentrale Umweltbelastungen finden, welche auch in anderen Branchen verwendet werden können. Eine ökologische Bewertung wird erlauben, die Anstrengungen bezüglich Aufwand (an Zeit und Finanzen) und ökologischem Return-on-Investment zu optimieren. Nur am Rande sei erwähnt, dass Ökobilanzen darüber hinaus eine ökologisch gezielte Führung eines Unternehmens entscheidend erleichtern oder sogar erst ermöglichen werden. Denn eindeutige und einheitliche ökologische Werte erlauben klare Zielvorgaben ('Mondlandung!') über einzelne Produkte hinaus. Auf der Basis einer umfassenden Ökobilanz können dann z.B. 'Umweltbudgets' ins Management by Objectives integriert werden, um nur ein Beispiel zu nennen.
Zusammenfassend gilt m.E., dass

- die effektive Entsorgung nicht unbedingt Aufgabe des Herstellers ist, wohl aber das Entsorgungsdesign eines Produktes;

- die optimale Entsorgbarkeit erreicht werden kann, indem - wie an Beispielen gezeigt - die Produktentwicklung den gesamten Produktlebenszyklus ins ökologische Kalkül einbezieht;

- eine umfassende ökologische Optimierung auch davon abhängt, klare ökologische Ziele setzen zu können. Dazu ist die Weiterentwicklung der Ökobilanz als betriebliches Führungsinstrument notwendig.

A. Braunschweig: Die Ökologische Buchhaltung in der städtischen Umweltpolitik, in: Dokumente und Informationen zur Schweiz. Orts-, Regional- und Landesplanung (DISP) Nr. 97/April 1989, ETH Hönggerberg Zürich, 1989.

BUWAL: Ökobilanzen von Packstoffen. Schriftenreihe Umweltschutz Nr. 24, Bern, 1984.

T. Dyllick: Ökologisch bewusste Unternehmensführung - Der Beitrag der Managementlehre. Ö.B.U.Schriftenreihe 1/1989. St. Gallen, 1989. 32 S.

J. Elkington, T. Burke: Umweltkrise als Chance. Okologische Herausforderung für die Industrie. Zürich, 1989.

R. Müller-Wenk: Die ökologische Buchhaltung. Frankfurt, 1978.

\title{
Reststoffentsorgung bei Kehrichtverbrennungsanlagen
}

\author{
Conrad M. Bader* und Jürg Wiedersheim
}

\section{Einleitung}

Abfall produzieren nicht nur Industrie und Gewerbe, die alles daran setzen, um diesen zu verringern. Abfälle produzieren auch die Haushalte, also wir alle. Und da in der Schweiz glücklicherweise heute $80 \%$ des Kehrichts in einer Kehrichtverbrennungsanlage beseitigt wird, müssen die beträchtlichen Mengen der dort anfallenden Reststoffe umweltverträglich entsorgt werden. Dieser Beitrag stellt die heute dazu bereits kommerziell verfügbaren Verfahren vor.

\section{Was sind die Reststoffe einer Kehrichtverbrennung?}

In der Schweiz stehen heute $36 \mathrm{Keh}$ richtsverbrennungsanlagen in Betrieb, die im Jahre 1988 insgesamt 2300000 t Kehricht entsorgt haben.

Die wesentliche Aufgabe der Kehrichtverbrennung besteht darin:

1. Masse und Volumen der Abfälle zu verringern,

2. durch Oxidation der Abfälle Schadstoffe soweit wie möglich zu zerstören,

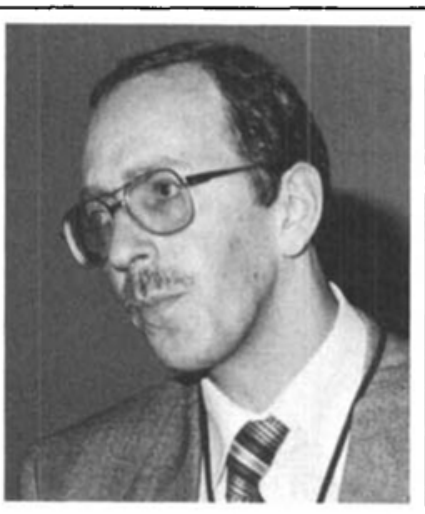

Conrad Bader: Geboren 1952, erwarb 1976 an der ETH Zürich sein Diplom als Maschineningenieur in Fachrichtung Verfahrenstechnik. Nach Tätigkeiten bei den Firmen Escher Wyss, Cora Engineering und Alfa Laval in den Bereichen Prozesstechnik und Marketing trat er 1987 als Assistent Marketing in den Bereich 'Trenn- und Mischverfahren' der Gebrüder Sulzer ein und leitet seit Mitte 1988 die Gruppe 'Umwelttechnik'. Diese entwickelt und baut Sondermüllverbrennungsanlagen, weitergehende Rauchgasreinigungen sowie Aufbereitungsanlagen für Reststoffe von Kehricht- und Sondermüllverbrennungsanlagen.

\footnotetext{
* Korrespondenz: C.M. Bader Gebrüder Sulzer $A G$

Umwelttechnik

Postfach

$\mathrm{CH}-840 \mathrm{l}$ Winterthur
} 

freizusetzen und soweit wie möglich zu nutzen, ren und in eine deponiefähige oder verwertbare Form zu bringen.

Zur Verbrennung von $1 \mathrm{t}$ Kehricht mit einem unteren Heizwert von $8500 \mathrm{~kJ} / \mathrm{kg}$ benötigt man etwa $5300 \mathrm{Nm}^{3}$ Luft und es entstehen ungefähr $300 \mathrm{~kg}$ Schlacke sowie $7800 \mathrm{~kg}$ Rauchgase. Letztere enthalten normalerweise 20-30 kg Flugstaub, 6-10 $\mathrm{kg}$ Salzsäure, 1-2 kg Schwefeldioxid, 1,5-2 $\mathrm{kg}$ Stickoxide sowie 150-400 g Kohlenwasserstoffe. Zusätzlich enthalten sind 50 g Flußsäure, $50 \mathrm{~g}$ Blei- und Zinkoxid sowie etwa 2 g Quecksilber (Fig. l).

Die schädlichen Schwermetalle werden hauptsächlich in der Schlacke und im Flugstaub zurückgehalten. Je besser die filternde Staubabscheidung funktioniert, be der heute schon vielfach $5-10 \mathrm{mg} / \mathrm{Nm}^{3} \mathrm{er}$ reicht werden, desto höher liegen die Schwermetallkonzentrationen im Flugstaub.
3. die in den Abfällen enthaltene Energie

4. verbleibende Reststoffe zu mineralisie-

Aus den 1988 in der Schweiz verbrannten $2300000 \mathrm{t}$ Kehricht entstanden als feste Rückstände $700000 \mathrm{t}$ trockene Schlacke, 50000 t Kessel- und Flugasche und ca. $10000 \mathrm{t}$ weitergehende Rauchgasreinigungsrückstände. Die Schadstoffverteilung auf diese drei Ströme präsentiert sich gemäss Fig. 2. Die Verbrennung von Kehricht führt zu einer Fraktionierung und Konzentrierung von Elementen, respektive ihren Verbindungen in einzelnen Reststoffen. Entsprechende Untersuchungen haben bestätigt, dass sich schwerflüchtige Metalle, vorwiegend Eisen und Chrom, in der Schlacke anreichern. Die leichtflüchtigen Metalle Quecksilber, Cadmium und Zink sowie Chloride und Sulfate treten bevorzugt im Flugstaub oder im Rauchgasreinigungsrückstand auf. Allgemein werden leichtlösliche Schwermetallchloride vorwiegend im Elektrofilterstaub und in der weitergehenden Rauchgasreinigung abgeschieden. Die schwerlöslichen Metalloxide verbleiben in der Schlacke.

Der Entwurf der Technischen Verordnung Abfall (TVA) sieht für diese Stoff-

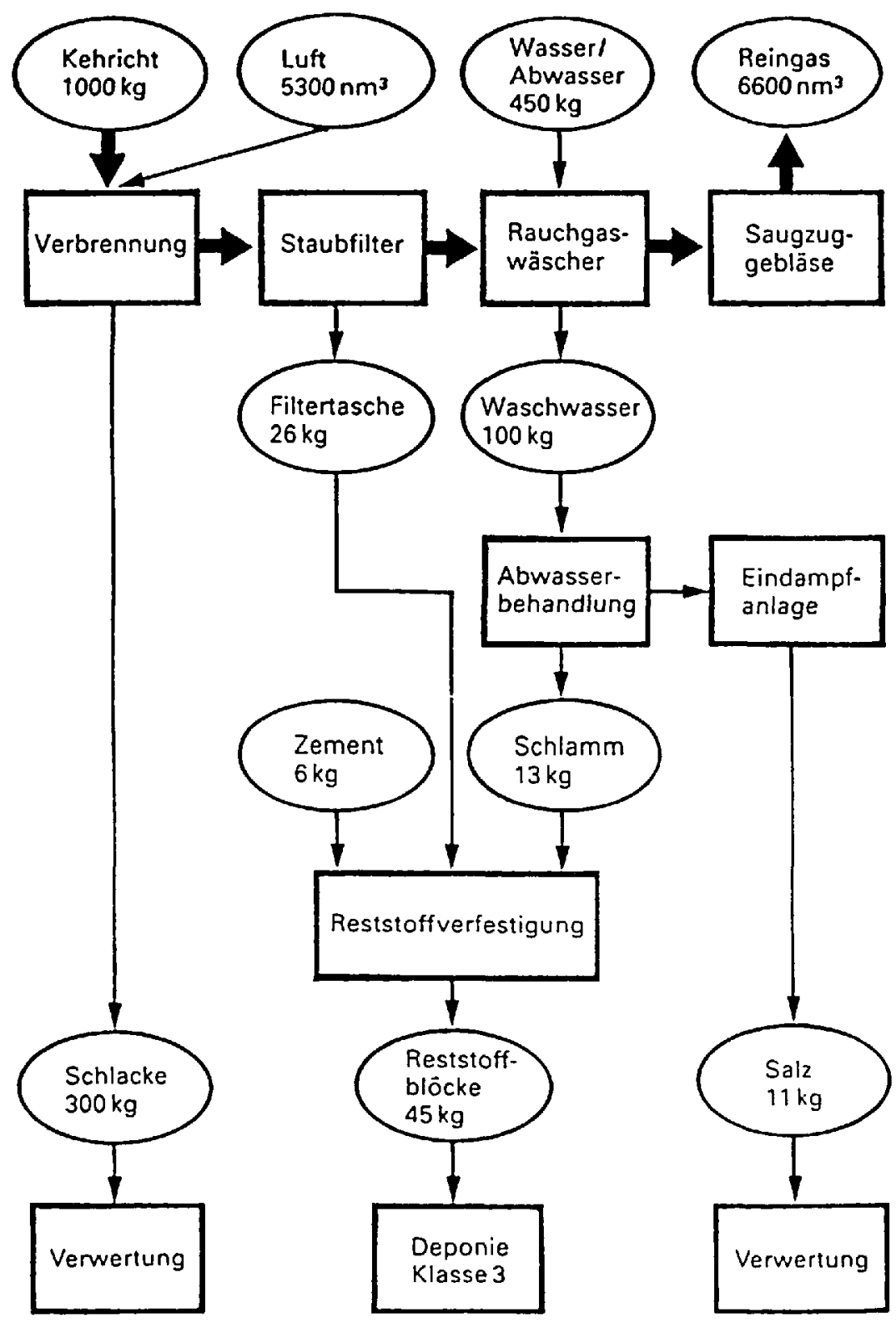

Fig. 1. Massenströme bei der Verbrennung von 1 t Kehricht ströme verschieden geartete Behandlungsresp. Entsorgungsmöglichkeiten vor:

\section{Schlacke}

Die Schlacke aus der Kehrichtverbrennung kann unter bestimmten Einschränkungen zum Bau von Strassen, Plätzen oder Dämmen eingesetzt werden, sofern folgende Anforderungen erreicht sind:

- der Glühverlust darf $2 \%$ Gewicht nicht übersteigen

- der Restgehalt an Chlorid darf nicht mehr als $1 \mathrm{~g} / \mathrm{kg}$ Schlacke betragen

- Schrott und grobe Teile sowie die Feinkornfraktion unter $0,1 \mathrm{~mm}$ sind abzutrennen

- die Schlacke ist beim Austritt aus dem Ofen zu benetzen und während mindestens einem Monat feucht zu lagern ('zu reifen')

Um diese Vorgaben zu erreichen, muss die Schlacke in einer aus weitgehend konventionellen Teilen bestehenden Anlage behandelt werden, wobei keinerlei besondere Probleme entstehen. Dass das bei dieser Aufarbeitung entstehende Wasch- und Sickerwasser auf Einleitqualität aufgearbeitet werden muss, versteht sich von selbst. Üblicherweise geschieht dies, gemeinsam mit dem Abwasser der weitergehenden Rauchgasreinigung und der Flugaschenwäsche, in einer Abwasserreinigungsanlage mit Schwermetallfällungsstufe.

Falls die Aufarbeitung der Schlacke nicht realisiert wird, besteht gemäss TVA auch die Möglichkeit, die unbehandelte Schlacke direkt in eine Reaktordeponie einzulagern. Dabei handelt es sich um eine kontrollierte Deponie für Abfälle mit mittlerem Schadstoffgehalt, bei der sowohl Deponiegas als auch Abwasser gefasst und behandelt werden müssen. Im übrigen bleibt anzumerken, dass die heute noch häufig praktizierte Vermischung von Schlacke, Kessel- und/oder Elektrofilterasche (Flugasche) gemäss der neuen TVA nicht mehr zulässig sein wird.

Flugstaub und Rückstände aus weitergehenden Rauchgasreinigungsanlagen

Im Flugstaub werden die Feststoffraktionen zusammengefasst, die im Kessel und im Elektrofilter aus dem Rauchgasstrom abgetrennt werden. Je nach Typ der verwendeten Rauchgasreinigungsanlage sind darin auch die Reaktionsprodukte der weitergehenden Rauchgasreinigung enthalten. Dies führt dazu, dass der Flugstaub von KVA zu KVA sehr unterschiedliche Eigenschaften aufweisen kann. In den folgenden Ausführungen werden die Bezeichnungen 'Flugstaub', 'Elektrofilterasche' sowie 'Reaktionsprodukt aus weitergehenden Rauchgasreinigungsanlagen' gleichwertig verwendet für die aus dem Kessel sowie dem Elektrofilter anfallenden Feststoffe. 


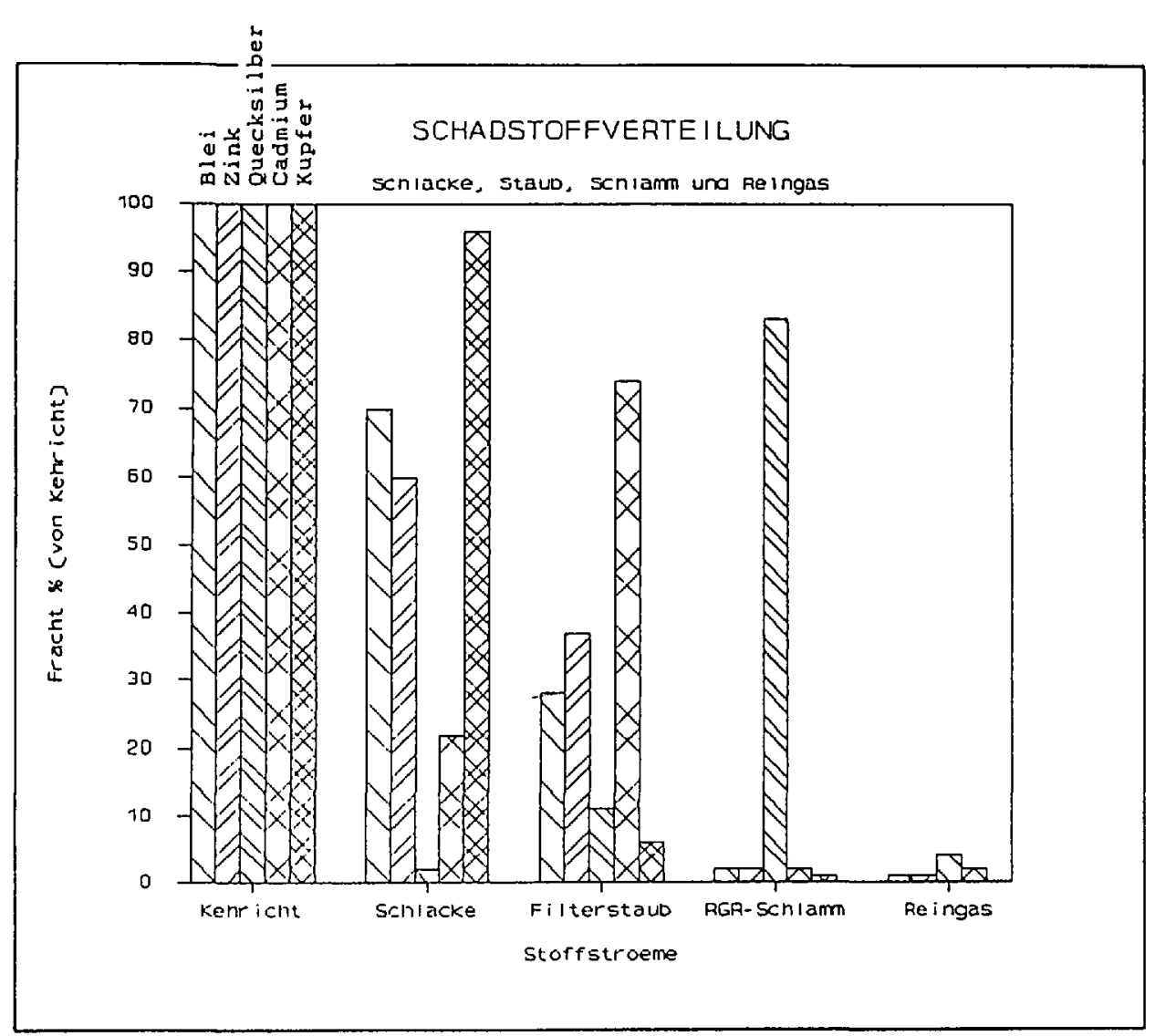

Fig. 2. Schadstoffverteilung in Abfall, Schlacke, Filterstaub und Rauchgasreinigungsschlamm

Bereits 1983 hat das Bundesamt für Umweltschutz (heute BUWAL) eine Arbeitsgruppe geschaffen, die sich intensiv mit den Voraussetzungen für eine umweltgerechte Entsorgung von Reststoffen aus Kehrichtverbrennungsanlagen auseinandersetzte. Im Rahmen der durchgeführten Arbeiten konnte gezeigt werden, dass sich das Auswaschen von Schwermetallen aus Elektrofilteraschen durch geeignete Vorbehandlung der Filterstäube drastisch reduzieren lässt. Die dabei gewonnenen Grundlagen wurden durch das Bundesamt für Umweltschutz in der Schriftenreihe Umweltschutz Band 62 publiziert.

Im Auftrag des Abfuhrwesens der Stadt Zürich hat anschliessend die Elektrowatt Ingenieurunternehmung $A G$ ein Verfahrenskonzept zur Aufarbeitung der Reststoffe aus Rauchgasreinigungsanlagen und die Grundlagen für eine Pilotanlage erarbeitet. Im Dezember 1986 erfolgte ein Auftrag an die Firma Sulzer zum Bau und Betrieb einer Pilotanlage durch eine Auftraggebergemeinschaft, der das BUS, die Kantone Zürich, Thurgau und St. Gallen, die Städte Zürich und Winterthur sowie der Zweckverband Kehrichtbeseitigung Linthgebiet angehörten. Im August 1987 wurde ein Zusatzauftrag erteilt, der die Erarbeitung von Verfestigungsrezepturen sowie die Evaluation und halbtechnische Erprobung von geeigneten Mischern umfasste.

\section{Konzept der Flugstaub-Aufbereitung}

Das Konzept der Flugstaub-Aufbereitung (Fig. 3) sieht in einem ersten Schritt die Extraktion der leicht wasserlöslichen, nichttoxischen Substanzen vor, in dem die Rückstände in einem bestimmten Verhältnis in Wasser suspendiert und während eimischt werden. Trotz sorgfältiger Kontrolle der Prozessparameter (Verweilzeit, $\mathrm{pH})$ lässt sich nicht verhindern, dass sich auch ein kleiner Teil der problematischen Substanzen, d.h. der Schwermetalle, im Wasser lösen. Nach Abschluss der eigentlichen Extraktion wird die Suspension filtriert. Durch zweimaliges Nachwaschen der entwässerten Rückstände auf der Filtrationsanlage wird erreicht, dass auch die im Mutterfiltrat enthaltenen leicht wasserlöslichen Substanzen aus den Rückständen verdrängt werden.

Die auf diese Weise vom wasserlöslichen Anteil befreiten Rückstände werden nun einem Zwangsmischer zugeführt, wo sie mit Zement und eventuell benötigten $\mathrm{Zu}$ schlagstoffen versetzt und homogen vermischt werden. Anschliessend erfolgt die Verdichtung der Mischung entweder durch Vibrieren oder über eine Hochdruckpresse zur Herstellung von Blöcken oder Pellets. Die Mischung kann auch unverdichtet in die Deponie eingebracht und erst an Ort verdichtet werden.

\section{Verfahrenstechnische Lösung}

Die Flugasche wird aus den Stapelsilos der KVA entweder mechanisch oder pneumatisch in einen Tagessilo befördert. Falls die Behandlung in einer zentralen Anlage erfolgt, ist der Aschetransport mit Silowaner definierten Verweilzeit intensiv ge- gen per Bahn oder Strasse vorgesehen. Mittels Zellradschleuse oder Austragsschnecke werden die Rückstände in den ersten Rührkesselreaktor unter gleichzeitiger Zugabe von Frischwasser dosiert. Dort wird ebenfalls die in der Abwasserreinigungsanlage anfallende Schlammsuspension zugegeben. Damit fält am Ende des ganzen Prozesses nur ein einziger Reststoff an, der bereits homogen gemischt ist. Durch die Regelung des $\mathrm{pH}$-Wertes auf $\mathrm{pH}$ 9,5-10 durch Säure oder Lauge wird erreicht, dass der Grossteil der Schwermetalle bereits während der Extraktion der leicht wasserlöslichen Substanzen als $\mathrm{Hy}$ droxide gefällt wird und somit im Rückstand verbleibt. Über einen Überlauf gelangt die Mischsuspension in einen zweiten Rührkesselreaktor. Eine Kaskade zur Extraktion des wasserlöslichen Anteils wurde gewählt, um die Verweilzeitverteilung einzuengen, was sich direkt auf die Filtrierbarkeit des Feststoffes auswirkt.

Eine totale mittlere Verweilzeit von 20 Minuten genügt vollauf zur Einstellung der Lösungsgleichgewichte. Mit einer Pumpe wird nun die Mischsuspension aus dem zweiten Extraktionsreaktor auf einen Vakuumbandfilter geführt, wo die Entwässerung vorgenommen wird. In zwei nachgeschalteten Waschstufen wird der Filterkuchen vom Mutterfiltrat befreit, dabei wird in der ersten Waschstufe als Medium das Bandwaschwasser verwendet. In der zweiten Waschstufe wird Frischwasser verwendet. Um einen höheren Gehalt an Trockensubstanz zu erreichen, wird der Filterkuchen zusätzlich gepresst. Wegen Gipsausfällungen hat sich das Waschen des Filterkuchens im Gegenstrom, das apparativ ohne grossen Aufwand möglich ist, in der Praxis nicht bewährt.

Trotz der Regelung des $\mathrm{pH}$-Wertes während der Extraktion der leicht wasserlöslichen Anteile kann nicht verhindert werden, dass ein kleiner Teil der Schwermetalle in Lösung bleiben, somit werden die vereinigten Filtrate der Abwasserreinigungsanlage zugeführt. Dort werden die Schwermetalle gefällt und über eine Membranfiltration oder eine Kammerfilterpresse separiert und in die Extraktionsstufe zurückgegeben.

Die auf diese Art und Weise aufbereiteten Rückstände enthalten bei einem Trokkensubstanzgehalt von $50-60 \%$ noch $0,5-$ $1,0 \%$ wasserlösliche Substanzen, womit der in der TVA geforderte Wert von maximal $5 \%$ massiv unterschritten wird. Zur eigentlichen Verfestigung der Rückstände werden sie zusammen mit dem hydraulischen Bindemittel und den eventuell benötigten Zuschlagstoffen in einen Zwangsmischer geführt und homogen gemischt. Das in den Rückständen aus dem Waschprozess noch vorhandene Wasser genügt vollauf für die Hydratation des Zementes.

Die standardisierte Flugascheaufbereitungsanlage (Fig. 4) kommt mit einem Minimum an Förderorganen aus, da der $\mathrm{Ma}$ terialfluss konsequent in Richtung der Schwerkraft erfolgt. Die Anlage enthält 
Rückstăndo von Rauchgasreinigung

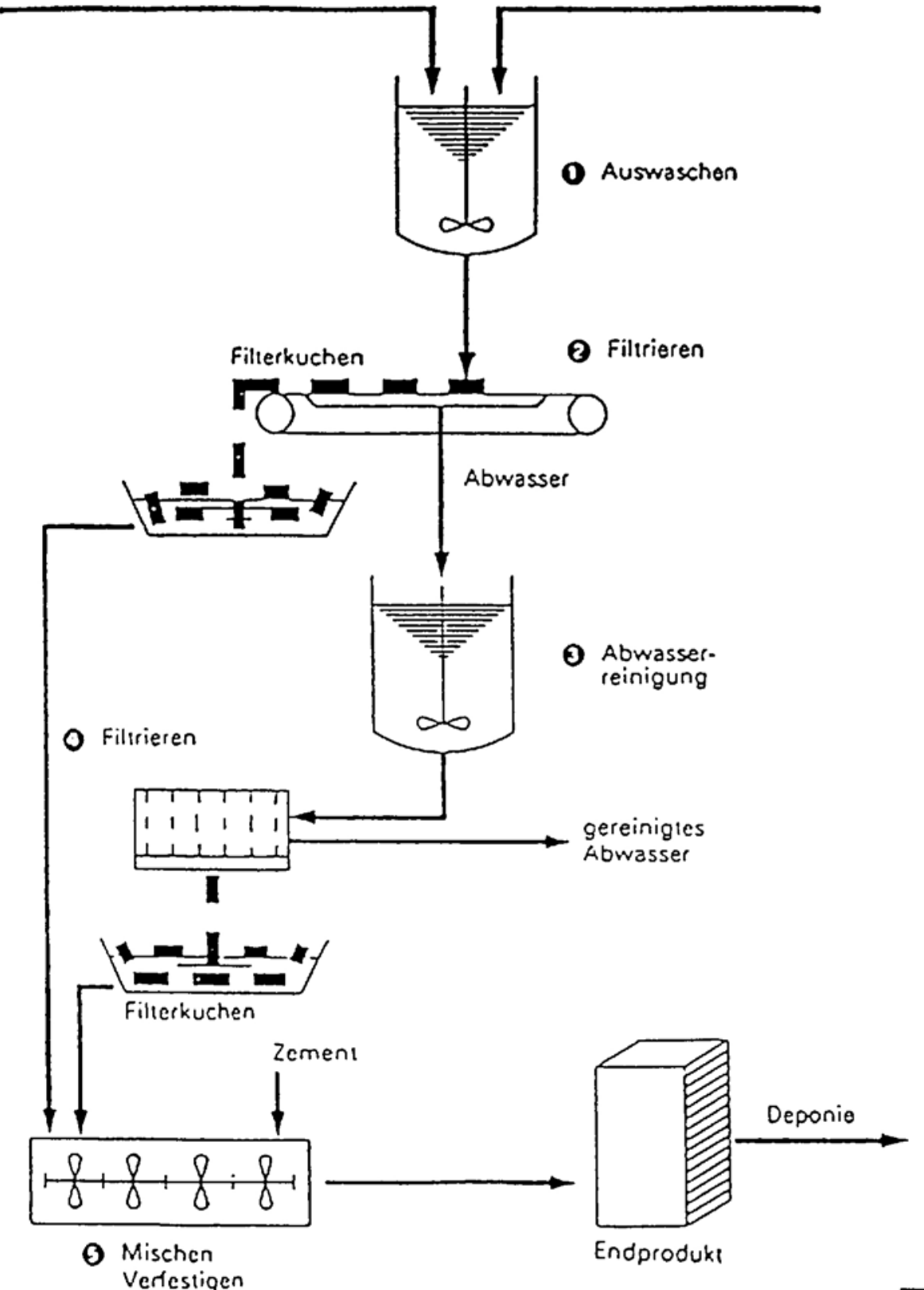

tiv hohen Wassergehalt voraussetzt. Dieser bewirkt eine Abbindezeit von bis zu mehreren Tagen. Entsprechend gross müssen die Lager für Formen und nicht ausgeschalte Blöcke dimensioniert werden. Bei der Herstellung von Blöcken durch mechanische Verdichtung sind für grössere Körper enorme Kräfte nötig, was einen grossen apparativen Aufwand bedingt.

\section{Herstellung von Pellets}

Reststoffpellets werden aus erdfeuchtem Material gepresst. Die Herstellung erfolgt auf bewährten Maschinen grosser Leistung. Da die Pellets sofort nach Verlassen der Pressen geschüttet werden, ist eine genügend grosse Festigkeit der Körper schon vor dem Abbinden des Zementes zwingende Voraussetzung für den problemlosen Betrieb einer Anlage. Dies kann durch eine optimierte Rezeptur und eine tiefe Restfeuchte der verarbeiteten Reststoffe erreicht werden. Das Einbringen der Pellets in die Deponie als Schüttung stellt keine Probleme. Es wird aber erwartet, dass, bedingt durch das geringere Schüttgewicht der Pellets im Vergleich zu Blökken, etwa $20 \%$ mehr Deponievolumen pro Tonne Reststoff benötigt wird.

\section{Direkter Einbau in die Deponie}

Der in-situ-Einbau der erdfeuchten Mischung mit anschliessender Verdichtung in der Deponie mit Walzen entspricht der

Fig. 3. Konzept Flugaschewäsche

alle beschriebenen Prozeßstufen. Die Lager für Flugasche, Zement und Reststoffblöcke müssen, den örtlichen Gegebenheiten angepasst, separat bereitgestellt werden.

\section{Verfestigung und Deponierung}

Zur Deponierung der Rückstände stehen folgende Techniken im Vordergrund:

- Herstellung von Blöcken

- Herstellung von Pellets

- Einbau der Zement-Rückstand-Mischung direkt in die Deponie

\section{Blockherstellung}

Blöcke aus Reststoffen können entweder in Formen gegossen und verdichtet oder auf Hochdruckpressen gepresst werden. Für das Vergiessen muss die Mischung plastisch vorliegen, was einen rela-

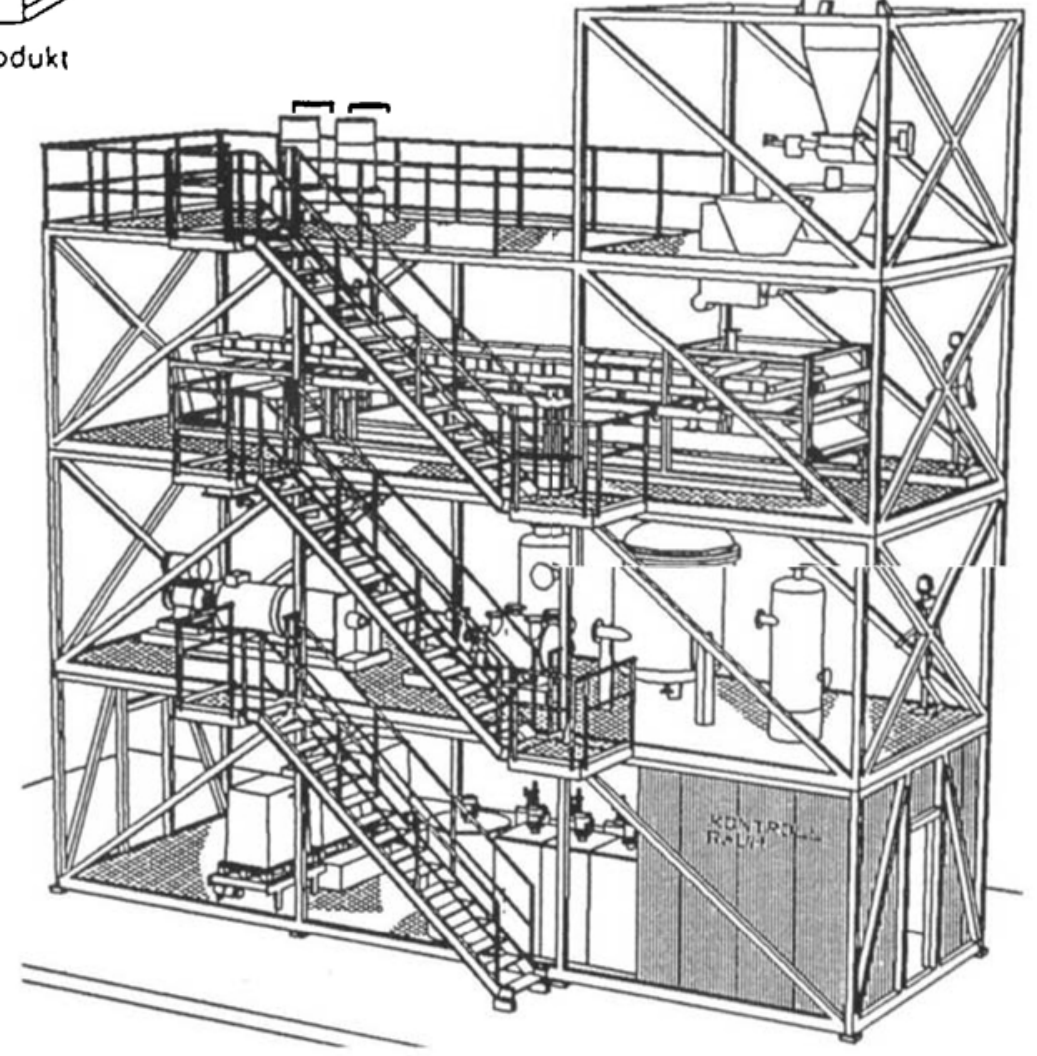

Fig. 4. Standardanlage Flugaschewäsche $1000 \mathrm{~kg} / \mathrm{h}$ 
heute üblichen Vorgehensweise bei Bodenverdichtungen. Diese Technik setzt voraus, dass sich die Deponie nahe am Mischort befindet, um ein Abbinden des Zementes in unverdichtetem Zustand auszuschliessen. Da der Einbau witterungsabhängig ist, sind relativ grosse Lager für gewaschene Rückstände erforderlich.

\section{Bewertung der verschiedenen Verfahren}

Im heutigen Zeitpunkt zeichnen sich noch keine klaren Präferenzen ab. Bei den verschiedenen in Planung und Bau befindlichen Anlagen in der Schweiz sind alle vorgestellten Varianten von Verfestigung anzutreffen. Die Wahl erfolgt weitgehend aufgrund der lokalen Gegebenheiten.

\section{Zusammenfassung}

Die aus der Kehrichtverbrennung anfallenden Reststoffe Schlacke, Flugstaub sowie Rückstände aus der weitergehenden Rauchgasreinigungsanlage können mit halbtechnisch bewährten Verfahren aufgearbeitet werden, so dass sie in einer der TVA gerechten Form vorliegen und entsorgt werden können. Die dank zielgerichteter Unterstützung der öffentlichen Hand bereits abgeschlossenen und noch laufenden Pilotprojekte erlaubten es, innert kurzer Zeit ein neues Verfahren vom Konzept zur Ausführungsreife zu bringen und somit den Betreibern von Kehrichtverbrennungsanlagen die Möglichkeit zu geben, die Auflagen der neuen TVA schon kurzfristig mit einem geeigneten Verfahren zu erfüllen.
Chimia 44 (1990) 201-202

(C) Schweiz. Chemiker-Verband; ISSN 0009-4293

\title{
Abfall, Materie am falschen Ort
}

\author{
(Titel oder Die Definition?)
}

\section{Hans Keller*}

Wenn Sie am Frühstückstisch die Butter statt auf das Brot versehentlich auf den Ärmel gestrichen haben, dann ist aus guter Butter Abfall geworden, resp. Sie haben Materie am falschen Ort.

Wird das Kleidungsstück dann noch mit Chlorkohlenwasserstoffen chemisch gereinigt, entsteht einer der schlimmsten Sonderabfälle. Wir wissen heute noch nicht recht, was man mit den CKW-Schlämmen aus der Kleiderreinigung anfangen soll.

Eben, um das Axiom von Lavoisier 'Rien ne se crée; Rien ne se perd' kommt man auch in der Bewirtschaftung des Abfalls nicht herum.

Unmöglich, 'ihn' zum Verschwinden zu bringen, indem man 'ihn unter den Teppich' wischt oder irgendwo in die Drittwelt verschifft.

Man tut gut daran, sich diese Grundbegriffe immer wieder in Erinnerung zu rufen. Jedes Atom, jedes Molekül, das ir-

\footnotetext{
* Korrespondenz: Dr. H. Keller

Cridec $S A$

$\mathrm{CH}-1013$ Eclépens
}

gendwo als Abfall auftritt, ist doch vorher irgendwo auf der Erde vorhanden gewesen.

Durch eine Veränderung, resp. durch eine Versetzung an einen andern Ort, ist es zum Abfall geworden.

Es muss uns gelingen, unseren Abfall entweder in eine erdkrustenähnliche Form zurückzuführen oder als Rohmaterial für neue Produkte zu verwenden. Materie, die sich am falschen Ort befindet, müssen wir wieder an den richtigen Ort bringen.

Mit anderen Worten, der menschliche Schaffungsgeist hat aus den Rohstoffen mittels Reaktionen und Transformationen Produkte synthetisiert. Werden diese Produkte $A b f a l l$, dann müssen wir 'entsynthetisieren' beziehungsweise 'recyclieren', d. $h$. einen geschlossenen Kreislauf herstellen.

Die Verfahren für diese Schritte in Richtung geschlossener Kreislauf müssen erarbeitet werden. Wir können uns nicht mehr erlauben, wertvolle Rohstoffe zu verarbeiten, ohne uns Gedanken darüber zu machen, was aus dem fabrizierten Gegenstand wird, wenn dieser als $A b f a l l$ anfällt.

Es gibt signifikante Beispiele solcher Problemlösungen. Zum Beispiel, bis vor
[1] Elektrowatt Ingenieurunternehmung $A G$ : Aufbereitung und Entsorgung von Reststoffen aus der Rauchgasreinigung von Kehrichtverbrennungsanlagen, Studie im Auftrag des Abfuhrwesens der Stadt Zürich, Zürich (März 1986).

[2] Bundesamt für Umweltschutz: Behandlung und Verfestigung von Rückständen aus Kehrichtverbrennungsanlagen, Schriftenreihe Umweltschutz, Nr. 62, Bern (April 1987).

[3] P. Baccini, P.H. Brunner, Behandlung und Endlagerung von Reststoffen aus Kehrichtverbrennungsanlagen; Gas-Wasser-Abwasser 1985, 65, p. 403/ 409.

[4] H.P. Tobler, Konzept zur Reststoffentsorgung in der Schweiz; VDI-Bericht 1989, 753, p. 9/33.

[5] U. Dietler, J. Wiedersheim, Aufarbeitung und Verfestigung von Rückständen der Rauchgasreinigung aus Kehrichtverbrennungsanlagen, 1988.

[6] EDI: Entwurf der Technischen Verordnung Abfall (TVA).

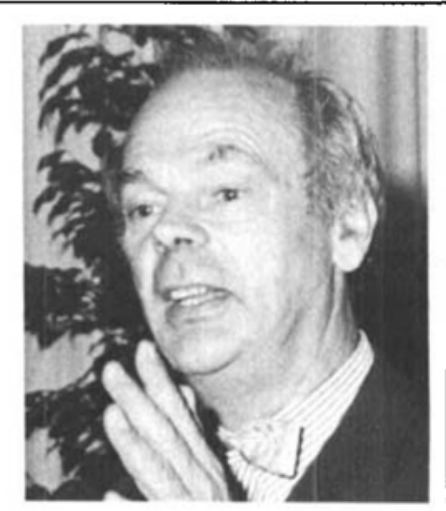

Hans Keller: Geboren 1927, im Aargau aufyewachsen. Beruf: Chemiker, Dr. Ing. Studium: Zürich und Genf, Universität; Dissertation in analytischer Chemie. Berufliche Tätigkeit: Analysen- u. Betriebslaboratorien der metallurgischen Industrie. (Stahlformung, Giesserei, Oberflächenbehandlung, Hableitertechnik). 25 Jahre als Angestellter in verschiedenen Industrien und dann weitere 9 Jahro als freischaffender Ingenieur der Material-Wissenschaft tätig. Neben Arbeiten in der Schweiz vielfach auch in Portugal, Ägypten, lran und speziel in Indien beschäftigt. Seit 4 Jahren verantwortlicher Leiter der Cridec SA (Centre de Ramassage et d'Identification des Déchets Spéciaux - Sammelstelle für Sonderabfälle). Zur Zeit auch sehr damit beschäftigt, einen Nachfolger zu finden.

wenigen Jahrzehnten wurde in der metallurgischen Industrie behauptet, dass mit ausrangierten Autos absolut nichts anzufangen sei. Es wurde argumentiert, das $\mathrm{Ge}-$ misch von Metallen und anderen Stoffen erlaube die Erfassung der einzelnen Rohstoffe nicht mehr. Und so hat man 'Autofriedhöfe' gefüllt, hektarenweise, und andere heute zum Teil fremd anmutende Problemlösungen angeboten. So wurden alte Autos im Meer versenkt mit der Begründung, die Fische hätten dann ein speziell gutes 'habitat'. 